\title{
Erratum: Three-body interactions improve contact prediction within direct-coupling analysis [Phys. Rev. E 96, 052405 (2017)]
}

\author{
Michael Schmidt and Kay Hamacher
}

(Received 10 June 2021; published 13 July 2021)

DOI: 10.1103/PhysRevE.104.019902

This article contains two typographical errors.

The first one is located in Sec. II B at the end of the paragraph and involves the equation for computation of the score $S_{i j}$. Each norm $\|\cdots\|$ in the formula has an erroneous square and the respective arguments should be the coupling matrices $\hat{\mathbf{J}}_{i j}$ in zero-sum gauge (indicated by the hat). The correct equation is

$$
S_{i j}=\left\|\hat{\mathbf{J}}_{i j}\right\|_{2}-\frac{\left\|\hat{\mathbf{J}}_{: j}\right\|_{2}\left\|\hat{\mathbf{J}}_{i:}\right\|_{2}}{\left\|\hat{\mathbf{J}}_{:: .}\right\|_{2}},
$$

and the norms read $\left\|\hat{\mathbf{J}}_{i j}\right\|_{2}=\sqrt{\sum_{l, m=1}^{q} \hat{J}_{i j}(l, m)^{2}}$.

The second typographical error is located in the $\hat{J}_{i j}$ calculation in Eq. (B5). The equation contains the erroneous term $\left[V_{i j k}^{\text {red }}(\mu(r), m, \eta)\right]$ with a missing mapping $\mu$ in the second argument. It should be $V_{i j k}^{\text {red }}(\mu(r), \mu(m), \eta)$. The corrected full Eq. (B5) is

$$
\begin{aligned}
\hat{J}_{i j}(l, m)= & J_{i j}(l, m)+\frac{1}{q} \sum_{r=1}^{q}\left[-J_{i j}(r, m)-J_{i j}(l, r)+\frac{1}{q} \sum_{s=1}^{q} J_{i j}(r, s)\right]+\frac{1}{q_{\mathrm{red}}} \sum_{\substack{k=1 \\
k \neq i, j}}^{N} \sum_{\eta=1}^{q_{\mathrm{red}}}\left[V_{i j k}^{\mathrm{red}}(\mu(l), \mu(m), \eta)\right. \\
& \left.+\frac{1}{q} \sum_{r=1}^{q}\left[-V_{i j k}^{\mathrm{red}}(\mu(r), \mu(m), \eta)-V_{i j k}^{\mathrm{red}}(\mu(l), \mu(r), \eta)+\frac{1}{q} \sum_{s=1}^{q} V_{i j k}^{\mathrm{red}}(\mu(r), \mu(s), \eta)\right]\right] .
\end{aligned}
$$

